\title{
Finite-Size Scaling Exponents of the Lipkin-Meshkov-Glick Model
}

\author{
Sébastien Dusuel ${ }^{1, *}$ and Julien Vidal ${ }^{2, \text { t }}$ \\ ${ }^{1}$ Institut für Theoretische Physik, Universität zu Köln, Zülpicher Str. 77, 50937 Köln, Germany \\ ${ }^{2}$ Groupe de Physique des Solides, CNRS UMR 7588, \\ Campus Boucicaut, 140 Rue de Lourmel, 75015 Paris, France
}

\begin{abstract}
We study the ground state properties of the critical Lipkin-Meshkov-Glick model. Using the Holstein-Primakoff boson representation, and the continuous unitary transformation technique, we compute explicitly the finite-size scaling exponents for the energy gap, the ground state energy, the magnetization, and the spin-spin correlation functions. Finally, we discuss the behavior of the two-spin entanglement in the vicinity of the phase transition.
\end{abstract}

PACS numbers: 75.40.Cx,05.10.Cc,11.10.Hi,03.65.Ud

Although Lipkin, Meshkov and Glick (LMG) introduced the model baring their name in nuclear physics [1], it is of much broader interest. It has thus been periodically revisited in different fields, such as statistical physics of spin systems [2, 3] or Bose-Einstein condensates [4] to cite only a few. More recently it has also drawn much attention in the quantum information framework, where it has been shown to display interesting entanglement properties [5, 6, 7, 8] different from those observed in one-dimensional models [9, 10]. After almost four decades, it has been proved to be integrable using algebraic Bethe ansatz [11, 12] or mapping it onto Richardson-Gaudin Hamiltonians for which exact solutions have been proposed [13]. However, although this integrability provides some important insights about the structure of the spectrum, it is useless to compute some physical quantities, such as the correlation functions, for a large number of degrees of freedom.

In the spin language that we shall adopt here, the LMG model describes mutually interacting spins half, embedded in a magnetic field. In the thermodynamical limit, it undergoes a quantum phase transition that is well described by a mean-field analysis. This transition can be first or second order depending whether the interaction is anti-ferromagnetic or ferromagnetic. In the latter case and at finite size, some nontrivial scaling behavior of observables have been found numerically [2, 3]. For instance, the energy gap seems to behave as $N^{-1 / 3}$ at the critical point where $N$ is the number of spins.

In this letter, we explicitly compute these finite-size scaling exponents combining a $1 / N$ expansion in the standard Holstein-Primakoff transformation, the continuous unitary transformations, and a scaling argument. First, we calculate the energy gap for which we detail the procedure. We also give the leading finite $N$ corrections for the ground state energy, the magnetization, and the spin-spin correlation functions. In a second step, we discuss the two-spin entanglement properties through the concurrence [14] which is directly related to these functions. These latter results are in excellent agreement with recent numerical studies [5, 15] which predict a cusp-like behavior of the concurrence at the transition point.
We consider the following Hamiltonian introduced by LMG 1]

$$
\begin{aligned}
H= & -\frac{\lambda}{N} \sum_{i<j}\left(\sigma_{x}^{i} \sigma_{x}^{j}+\gamma \sigma_{y}^{i} \sigma_{y}^{j}\right)-h \sum_{i} \sigma_{z}^{i} \\
= & -\frac{2 \lambda}{N}\left(S_{x}^{2}+\gamma S_{y}^{2}\right)-2 h S_{z}+\frac{\lambda}{2}(1+\gamma) \\
= & -\frac{\lambda}{N}(1+\gamma)\left(\mathbf{S}^{2}-S_{z}^{2}-N / 2\right)-2 h S_{z} \\
& -\frac{\lambda}{2 N}(1-\gamma)\left(S_{+}^{2}+S_{-}^{2}\right),
\end{aligned}
$$

where the $\sigma_{\alpha}$ 's are the Pauli matrices, $S_{\alpha}=\sum_{i} \sigma_{\alpha}^{i} / 2$, and $S_{ \pm}=S_{x} \pm i S_{y}$. The $1 / N$ prefactor ensures that the free energy per spin is finite in the thermodynamical limit. Here we focus on the ferromagnetic case $(\lambda>0)$ and all our results are valid for $|\gamma| \leq 1$ (the case $|\gamma|>$ 1 being trivially obtained by a simple rescaling of $\lambda$ ). In this situation, the Hamiltonian (3) displays a secondorder quantum phase transition at $\lambda=|h|[2,3]$. In the sequel, we restrict our discussion to the phase $|h| \geq \lambda$, and without loss of generality, we set $h=1$.

The Hamiltonian $H$ preserves the magnitude of the total spin and does not couple states having a different parity of the number of spins pointing in the magnetic field direction (spin-flip symmetry), namely

$$
\left[H, \mathbf{S}^{2}\right]=0, \quad \text { and } \quad\left[H, \prod_{i} \sigma_{z}^{i}\right]=0
$$

for all values of the anisotropy parameter $\gamma$. In the isotropic case $\gamma=1$, one further has $\left[H, S_{z}\right]=0$, so that $H$ is diagonal in the eigenbasis of $\mathbf{S}^{2}$ and $S_{z}$. Due to the ferromagnetic interaction between the spins, the ground state and the first excited state always lie in the subspace of maximum spin $S=N / 2$.

In order to analyze the spectrum of $H$ in the large $N$ limit and to capture the finite-size corrections, we perform a $1 / N$ expansion of the low-energy spectrum, following the ideas of Stein [16]. We first use the HolsteinPrimakoff boson representation of the spin operator 17] 
in the $S=N / 2$ subspace given by

$$
\begin{aligned}
& S_{z}=S-a^{\dagger} a=N / 2-a^{\dagger} a, \\
& S_{+}=\left(2 S-a^{\dagger} a\right)^{1 / 2} a=N^{1 / 2}\left(1-a^{\dagger} a / N\right)^{1 / 2} a, \\
& S_{-}=a^{\dagger}\left(2 S-a^{\dagger} a\right)^{1 / 2}=N^{1 / 2} a^{\dagger}\left(1-a^{\dagger} a / N\right)^{1 / 2}
\end{aligned}
$$

where the standard bosonic creation and annihilation operators satisfy $\left[a, a^{\dagger}\right]=1$. This representation is well adapted to the computation of the low-energy physics with $\left\langle a^{\dagger} a\right\rangle / N \ll 1$. After inserting these latter expressions of the spin operators in Eq. (3), one expands the square roots in their Taylor series, and writes the result in normal ordered form with respect to the zero boson state. The Hamiltonian then reads $H=H_{0}+H_{2}^{+}+H_{2}^{-}$, with

$$
H_{0}=\sum_{\alpha, \delta \in \mathbb{N}} \frac{h_{0, \alpha}^{(\delta)} A_{\alpha}}{N^{\alpha+\delta-1}} \text { and } H_{2}^{+}=\sum_{\alpha, \delta \in \mathbb{N}} \frac{h_{2, \alpha}^{(\delta)} a^{\dagger^{2}} A_{\alpha}}{N^{\alpha+\delta}},
$$

and with $H_{2}^{-}=\left(H_{2}^{+}\right)^{\dagger}$ and $A_{\alpha}=a^{\dagger^{\alpha}} a^{\alpha}$. The index $\alpha$ keeps track of the number of bosonic operators, and for a given $\alpha$, the superscript $\delta$ codes the successive $1 / N$ corrections. For instance, the nonvanishing coefficients of $H_{0}$ are given by $h_{0,0}^{(0)}=-1, h_{0,0}^{(1)}=0, h_{0,1}^{(0)}=2-\lambda(1+\gamma)$, $h_{0,1}^{(1)}=\lambda(1+\gamma)$ and $h_{0,2}^{(0)}=\lambda(1+\gamma)$.

Next, the Hamiltonian is diagonalized order by order in $1 / N$ using the continuous unitary transformation method, introduced by Wegner [18] and independently by Głazek and Wilson [19, 20]. For a pedagogical introduction to this technique, see Ref. 21]. Note that the method has been applied to the LMG model in [22, 23, 24, 25], but its simultaneous use with the $1 / N$ expansion originates in [16]. The main idea is to diagonalize the Hamiltonian in a continuous way starting from the original (bare) Hamiltonian $H=H(l=0)$. A flowing Hamiltonian is then defined by

$$
H(l)=U^{\dagger}(l) H U(l),
$$

where $l$ is a scaling parameter such that $H(l=\infty)$ is diagonal. A derivation of Eq. (9) with respect to $l$ yields the flow equation

$$
\partial_{l} H(l)=[\eta(l), H(l)] \text {, where } \eta(l)=-U^{\dagger}(l) \partial_{l} U(l) .
$$

The anti-hermitian generator $\eta(l)$ must be chosen to bring the final Hamiltonian to a diagonal form. Wegner proposed $\eta(l)=\left[H_{\mathrm{d}}(l), H_{\mathrm{od}}(l)\right]=\left[H_{\mathrm{d}}(l), H(l)\right]$, where $H_{\mathrm{d}}$ and $H_{\mathrm{od}}$ are the diagonal and off-diagonal parts of the Hamiltonian. For our problem, it would read $\eta(l)=\left[H_{0}(l), H_{2}^{+}(l)+H_{2}^{-}(l)\right]$. Such a choice suffers from the drawback that the tridiagonality of $H(l=0)$ is lost during the flow and that $H(l)$ contains some terms which create any even number of excitations. This problem can be circumvented using the so-called quasi-particle conserving generator $\eta(l)=H_{2}^{+}(l)-H_{2}^{-}(l)$ that we shall use here. This generator was first proposed in 22, 26] and given a deeper physical meaning in 27].

More generally, to compute the expectation value of any operator $\Omega$ on an eigenstate $|\psi\rangle$ of $H$ with eigenvalue $E$, one must follow the flow of the operator $\Omega(l)=$ $U^{\dagger}(l) \Omega U(l)$, by solving $\partial_{l} \Omega(l)=[\eta(l), \Omega(l)]$. Indeed, one has:

$$
\langle\psi|\Omega| \psi\rangle=\left\langle\psi\left|U(l=\infty) \Omega(l=\infty) U^{\dagger}(l=\infty)\right| \psi\right\rangle,
$$

where $U^{\dagger}(l=\infty)|\psi\rangle$ is simply the eigenstate of the diagonal Hamiltonian $H(l=\infty)$ with eigenenergy $E$. In principle, one should follow the evolution of the $S_{x}, S_{y}$ and $S_{z}$ observables, from which all others can be deduced. However, since we aim at computing the ground state magnetization and spin-spin correlation functions, and because of the symmetries of the model, the calculation can be performed more simply as follows. First, the spinflip symmetry (4) implies

$$
\begin{aligned}
\left\langle S_{x}\right\rangle & =\left\langle S_{y}\right\rangle=0 \\
\left\langle S_{x} S_{z}\right\rangle=\left\langle S_{z} S_{x}\right\rangle & =\left\langle S_{y} S_{z}\right\rangle=\left\langle S_{z} S_{y}\right\rangle=0 .
\end{aligned}
$$

Furthermore, since the maximum spin representation is one-dimensional, the coefficients of the eigenstates in this sector can be chosen to be real so that $\left\langle\left\{S_{x}, S_{y}\right\}\right\rangle=0$. We are thus led to consider only the following (extensive) observables : $2 S_{z}, 4 S_{x}^{2} / N, 4 S_{y}^{2} / N$ and $4 S_{z}^{2} / N$. Of course, the structure of the flowing observables does not remain as simple as those of the initial conditions of the flow, even with our choice of the generator. In a notation similar to (8), all these observables can be written as $\Omega=\Omega_{0}+\sum_{k}\left(\Omega_{k}^{+}+\Omega_{k}^{-}\right)$, where the sum runs over all non-negative even integers $k$ 's, and

$$
\Omega_{0}=\sum_{\alpha, \delta \in \mathbb{N}} \frac{\omega_{0, \alpha}^{(\delta)} A_{\alpha}}{N^{\alpha+\delta-1}} \text { and } \Omega_{k}^{+}=\sum_{\alpha, \delta \in \mathbb{N}} \frac{\omega_{k, \alpha}^{(\delta)} a^{\dagger} A_{\alpha}}{N^{\alpha+\delta+k / 2-1}} .
$$

We have omitted the dependence on the flow parameter $l$ of the $\omega$ 's which is implicit in the following. For instance, the initial conditions for $2 S_{z}$ are $\omega_{0,0}^{(0)}=1, \omega_{0,1}^{(0)}=-2$, with all other coefficients vanishing.

The commutators $[\eta, H]$ and $[\eta, \Omega]$ are computed using $\left[a, a^{\dagger}\right]=1$ and basic counting results, yielding the flows

$$
\begin{aligned}
\partial_{l} h_{0, \alpha}^{(\delta)} & =2 \sum_{n, \alpha^{\prime}, \delta^{\prime}} \mathcal{A}_{\alpha^{\prime}, \alpha-\alpha^{\prime}-2+n}^{0, n} h_{2, \alpha^{\prime}}^{\left(\delta^{\prime}\right)} h_{2, \alpha-\alpha^{\prime}-2+n}^{\left(\delta-\delta^{\prime}+1-n\right)}, \\
\partial_{l} h_{2, \alpha}^{(\delta)} & =\sum_{n, \alpha^{\prime}, \delta^{\prime}} \mathcal{B}_{\alpha^{\prime}, \alpha-\alpha^{\prime}+n}^{0, n} h_{2, \alpha^{\prime}}^{\left(\delta^{\prime}\right)} h_{0, \alpha-\alpha^{\prime}+n}^{\left(\delta-\delta^{\prime}+1-n\right)}, \\
\partial_{l} \omega_{0, \alpha}^{(\delta)}= & 2 \sum_{n, \alpha^{\prime}, \delta^{\prime}} \mathcal{A}_{\alpha^{\prime}, \alpha-\alpha^{\prime}-2+n}^{0, n} h_{2, \alpha^{\prime}}^{\left(\delta^{\prime}\right)} \omega_{2, \alpha-\alpha^{\prime}-2+n}^{\left(\delta-\delta^{\prime}+1-n\right)}, \\
\partial_{l} \omega_{k, \alpha}^{(\delta)}= & \sum_{n, \alpha^{\prime}, \delta^{\prime}} h_{2, \alpha^{\prime}}^{\left(\delta^{\prime}\right)}\left[\mathcal{A}_{\alpha^{\prime}, \alpha-\alpha^{\prime}-2+n}^{k, n} \omega_{k+2, \alpha-\alpha^{\prime}-2+n}^{\left(\delta-\delta^{\prime}+1-n\right)}\right. \\
& \left.+\mathcal{B}_{\alpha^{\prime}, \alpha-\alpha^{\prime}+n}^{k-2, n} \omega_{k-2, \alpha-\alpha^{\prime}+n}^{\left(\delta-\delta^{\prime}+1-n\right)}\right],
\end{aligned}
$$


with the definitions

$$
\begin{aligned}
& \mathcal{A}_{\alpha^{\prime}, \alpha^{\prime \prime}}^{k, n}=n !\left(C_{\alpha^{\prime}}^{n} C_{\alpha^{\prime \prime}}^{n}-C_{\alpha^{\prime}+2}^{n} C_{\alpha^{\prime \prime}+k+2}^{n}\right), \\
& \mathcal{B}_{\alpha^{\prime}, \alpha^{\prime \prime}}^{k, n}=n !\left(C_{\alpha^{\prime}}^{n} C_{\alpha^{\prime \prime}+k}^{n}-C_{\alpha^{\prime}+2}^{n} C_{\alpha^{\prime \prime}}^{n}\right),
\end{aligned}
$$

$C_{\alpha}^{n}$ being the binomial coefficient $\alpha ! /[n !(\alpha-n) !]$. The sums in (15 18) are constrained by the fact that all subscripts and superscripts have to be positive. For example, in (15), $n$ runs from 0 to $1+\delta, \alpha^{\prime}$ from 0 to $\alpha-2+n$ and $\delta^{\prime}$ from 0 to $\delta+1-n$. At lowest nontrivial order in $1 / N$, Eqs. (1516) become

$$
\begin{aligned}
& \partial_{l} h_{0,0}^{(1)}=-4 h_{2,0}^{(0)^{2}} \\
& \partial_{l} h_{0,1}^{(0)}=-8 h_{2,0}^{(0)^{2}} \\
& \partial_{l} h_{2,0}^{(0)}=-2 h_{0,1}^{(0)} h_{2,0}^{(0)} .
\end{aligned}
$$

These equations are the well-known Bogoliubov transform, written in a differential form [21].

In 16], Stein integrated analytically the flow equations (15) and (16) for $\gamma=-1$, and computed the $1 / N$ corrections to the ground state energy and to the gap. For our purpose, we have generalized his solution to any value of $\gamma$, and went to order $1 / N^{3}$. Furthermore, we also computed the exact solutions to (17) and (18) and computed the $1 / N^{2}$ corrections to the extensive observables. Detailed calculations will be presented elsewhere [28].

To keep the presentation short, we will here only deal with the results obtained for the energy gap $\Delta$. We found

$$
\begin{aligned}
& \frac{\Delta(N)}{\Delta(\infty)}=1+\frac{1}{N}\left[\frac{P_{1}(\lambda, \gamma)}{\Xi(\lambda, \gamma)^{3 / 2}}+\frac{Q_{1}(\lambda, \gamma)}{\Xi(\lambda, \gamma)}\right] \\
& +\frac{1}{N^{2}}(1-\gamma)^{2}\left[\frac{P_{2}(\lambda, \gamma)}{\Xi(\lambda, \gamma)^{3}}+\frac{Q_{2}(\lambda, \gamma)}{\Xi(\lambda, \gamma)^{5 / 2}}\right] \\
& +\frac{1}{N^{3}}(1-\gamma)^{2}\left[\frac{P_{3}(\lambda, \gamma)}{\Xi(\lambda, \gamma)^{9 / 2}}+\frac{Q_{3}(\lambda, \gamma)}{\Xi(\lambda, \gamma)^{4}}\right]+\mathcal{O}\left(\frac{1}{N^{4}}\right),
\end{aligned}
$$

where $\Xi(\lambda, \gamma)=(1-\lambda)(1-\gamma \lambda), \Delta(\infty)=2 \Xi(\lambda, \gamma)^{1 / 2}$ is the mean-field gap [2, 3], and the $P_{i}$ 's and $Q_{i}$ 's are polynomial functions of $\lambda$ and $\gamma$. For the isotropic case $\gamma=1$, one has $P_{1}(\lambda, 1)=4 \lambda(1-\lambda)^{2}, Q_{1}(\lambda, 1)=-2 \lambda(1-$ $\lambda)$ and all contributions of order higher than $1 / N$ vanish, so that we recover the exact result $\Delta_{\gamma=1}(N)=2(1-\lambda)+$ $2 \lambda / N$.

Let us now discuss the case $\gamma<1$ for which we have checked that $\lambda=1$ is neither a root of the $P_{i}$ 's nor of the $Q_{i}$ 's. The result (24) shows that all $1 / N^{i}$ corrections diverge when $\lambda$ approaches the critical value 1 in the infinite system, such that the larger values of $i$, the stronger the divergence. However, physical quantities cannot display any singularity at finite $N$. Using the usual ideas of finite-size scaling 29] generalized in [2, 3] to infinitely coordinated systems, we can thus compute the scaling critical exponents. To this end, let us suppose $\lambda$ close to its critical value 1 . One can then neglect all $Q$ terms which are less divergent than the $P$ ones. In this limit, expression (24) becomes a function of the variable $N \Xi(\gamma, \lambda)^{3 / 2}$, namely

$$
\Delta(N) \simeq \Delta(\infty) \mathcal{F}_{\Delta}\left[N \Xi(\gamma, \lambda)^{3 / 2}, \gamma\right], \text { for } \lambda \simeq 1 .
$$

Thus, the scaling function $\mathcal{F}_{\Delta}$ for the gap must behave as $\left[N \Xi(\gamma, \lambda)^{3 / 2}\right]^{-1 / 3}$ in the vicinity of the critical point $\lambda=1$, for its product with the mean-field gap to be nonsingular. Consequently, one gets $\Delta(N) \sim N^{-1 / 3}$. Of course we have only checked the scaling hypothesis up to the order $1 / N^{3}$, but the integrability of the LMG model leads us to conjecture that the very simple structure of the $1 / N$ expansion exhibited in (24) is the same at all orders.

We have performed the same analysis for the ground state energy, the magnetization and the two-spin correlation functions for the ground state. All results are summarized below and detailed calculations will be presented in a forthcoming publication [28].

$$
\begin{aligned}
\Delta(N) & \sim a_{\Delta} N^{-1 / 3} \\
e_{0}(N) & \sim-1-(1-\gamma) /(2 N)+a_{e} N^{-4 / 3} \\
2\left\langle S_{z}\right\rangle / N & \sim 1+1 / N+a_{z} N^{-2 / 3} \\
4\left\langle S_{x}^{2}\right\rangle / N^{2} & \sim a_{x x} N^{-2 / 3} \\
4\left\langle S_{y}^{2}\right\rangle / N^{2} & \sim a_{y y} N^{-4 / 3} \\
4\left\langle S_{z}^{2}\right\rangle / N^{2} & \sim 1+2 / N+a_{z z} N^{-2 / 3}
\end{aligned}
$$

where $e_{0}$ denotes the ground state energy per spin. In each of the above expressions, we have first written the (exact) non-singular contributions and, second, the term coming from the resummation of the most singular terms in the $1 / N$ expansion. Let us note however that in (28) and (31), the $N^{-2 / 3}$ terms dominate the large $N$ behavior. The coefficients $a$ 's are real numbers that cannot be computed within our approach since the scaling argument only provides the exponents. Nevertheless, let us note that $a_{z z}=-a_{x x}$ since for all $N$, one has

$$
\frac{4}{N^{2}}\left(\left\langle S_{x}^{2}\right\rangle+\left\langle S_{y}^{2}\right\rangle+\left\langle S_{z}^{2}\right\rangle\right)=\frac{4 \mathbf{S}^{2}}{N^{2}}=1+\frac{2}{N} .
$$

As $\left\langle S_{x}^{2}\right\rangle$ is positive, one must also have $a_{x x} \geq 0$. One can furthermore infer that $N^{-4 / 3}$ corrections must exist in $4\left\langle S_{x}^{2}\right\rangle / N^{2}$ and/or in $4\left\langle S_{z}^{2}\right\rangle / N^{2}$, to cancel the one of $4\left\langle S_{y}^{2}\right\rangle / N^{2}$ in Eq. (32).

These results are in excellent agreement with the numerical data [3] where the exponents for $\Delta$ and for $4\left\langle S_{x}^{2}\right\rangle / N^{2}$ were conjectured. However, the scaling exponent $2 / 3$ for $2\left\langle S_{z}\right\rangle / N$ differs from that found in [15] $(0.55 \pm 0.01)$. This discrepancy comes from the too small system size investigated in 15$](N=500$ spins). We have indeed performed a numerical study up to $N=2^{14}$ spins and checked that the large $N$ leading exponent is indeed $2 / 3$ [28]. 
The finite-size scaling of the correlation functions also allows us to discuss the entanglement properties of the critical LMG model which have been the subject of several studies 5, 6, 7]. For the ferromagnetic case considered here, it has been shown numerically that the twospin entanglement, as measured by the (rescaled) concurrence [14], displays a singularity at $\lambda=1$. Actually, as shown by Wang and Mølmer [30], the concurrence $C$ for symmetric spin systems can be simply expressed in terms of the spin-spin correlation functions. More precisely, for the present case, one has

$$
(N-1) C=\frac{2}{N}\left(\left|\left\langle S_{x}^{2}-S_{y}^{2}\right\rangle\right|-N^{2} / 4+\left\langle S_{z}^{2}\right\rangle\right) .
$$

At the critical point, using the results (2930) and $a_{x x} \geq 0$, one can deduce that $\left\langle S_{x}^{2}-S_{y}^{2}\right\rangle$ is positive. Then using (32) and (30) one gets:

$$
(N-1) C_{\lambda=1}=1-\frac{4\left\langle S_{y}^{2}\right\rangle}{N} \sim 1-a_{y y} N^{-1 / 3} .
$$

This behavior is in agreement with the numerical study of the finite-size scaling presented in 5, 15]. In the thermodynamical limit $(N \rightarrow \infty)$, and in the phase $\lambda<1$, the Bogoliubov transform (21,23) also gives [28]

$$
\lim _{N \rightarrow \infty}(N-1) C_{\lambda<1}=1-\sqrt{\frac{1-\lambda}{1-\gamma \lambda}},
$$

which generalizes to any anisotropy parameter the expression recently given by Reslen et al. [15] for $\gamma=0$.

In summary, we have used the Holstein-Primakoff transformation and the continuous unitary transformations to analyze the finite size corrections of several observables in the LMG model. Using a $1 / N$ expansion and simple scaling arguments, we have captured nontrivial exponents that had been conjectured since several decades (see e.g. [2, 3]) but had never found any analytical support. This powerful combination of both methods clearly opens many routes to investigate. In principle, the physics of the broken phase $(\lambda>1)$ could also be tackled using the same approach, after performing a rotation bringing the $z$-axis along one of the two directions of the classical magnetization. However, in this phase, the gap, for instance, is known to behave like $\exp (-a N)$ 31] and may not be extracted from a $1 / N$ expansion. $A$ contrario, the behavior of the other quantities discussed here should be computed along the same line.

Finally, we wish to underline that the results presented here are also relevant for the Dicke model 32]. Indeed, in the zero temperature limit, the LMG model can be put in a one-to-one correspondence with this latter model as recently shown in [15].

We are indebted to B. Douçot, J. Dukelsky, S. Kirschner, D. Mouhanna, E. Müller-Hartmann, A. Reischl, A. Rosch and K. P. Schmidt for fruitful and valuable discussions. Financial support of the DFG in SP1073 is gratefully acknowledged.

* Electronic address: sdusuel@thp.uni-koeln.de

† Electronic address: vidal@gps.jussieu.fr

[1] H. J. Lipkin, N. Meshkov, and A. J. Glick, Nucl. Phys. 62, 188 (1965), N. Meshkov, A. J. Glick, and H. J. Lipkin, Nucl. Phys. 62, 199 (1965), A. J. Glick, H. J. Lipkin, and N. Meshkov, Nucl. Phys. 62, 211 (1965).

[2] R. Botet, R. Jullien, and P. Pfeuty, Phys. Rev. Lett. 49, 478 (1982).

[3] R. Botet and R. Jullien, Phys. Rev. B 28, 3955 (1983).

[4] J. I. Cirac, M. Lewenstein, K. Mølmer, and P. Zoller, Phys. Rev. A 57, 1208 (1998).

[5] J. Vidal, G. Palacios, and R. Mosseri, Phys. Rev. A 69, 022107 (2004).

[6] J. Vidal, R. Mosseri, and J. Dukelsky, Phys. Rev. A 69, 054101 (2004).

[7] J. Vidal, G. Palacios, and C. Aslangul, cond-mat/0406481

[8] J. I. Latorre, R. Orús, E. Rico, and J. Vidal, cond-mat/0409611

[9] T. J. Osborne and M. A. Nielsen, Phys. Rev. A 66, 032110 (2002).

[10] A. Osterloh, L. Amico, G. Falci, and R. Fazio, Nature (London) 416, 608 (2002).

[11] F. Pan and J. P. Draayer, Phys. Lett. B 451, 1 (1999).

[12] J. Links, H.-Q. Zhou, R. H. McKenzie, and M. D. Gould, J. Phys. A 36, R63 (2003).

[13] J. Dukelsky, S. Pittel, and G. Sierra, Rev. Mod. Phys. 76, 643 (2004).

[14] W. K. Wootters, Phys. Rev. Lett. 80, 2245 (1998).

[15] J. Reslen, L. Quiroga, and N. F. Johnson, quant-phys/0406674

[16] J. Stein, J. Phys. G 26, 377 (2000).

[17] T. Holstein and H. Primakoff, Phys. Rev. 58, 1098 (1940).

[18] F. Wegner, Ann. Physik 3, 77 (1994).

[19] S. D. Głazek and K. G. Wilson, Phys. Rev. D 48, 5863 (1993).

[20] S. D. Głazek and K. G. Wilson, Phys. Rev. D 49, 4214 (1994).

[21] S. Dusuel and G. S. Uhrig, J. Phys. A 37, 9275 (2004).

[22] A. Mielke, Eur. Phys. J. B 5, 605 (1998).

[23] H. J. Pirner and B. Friman, Phys. Lett. B 434, 231 (1998).

[24] F. G. Scholtz, B. H. Bartlett, and H. B. Geyer, Phys. Rev. Lett. 91, 080602 (2003).

[25] J. N. Kriel, A. Y. Morozov, and F. G. Scholtz, cond-mat/0408420

[26] J. Stein, Eur. Phys. J. B 5, 193 (1998).

[27] C. Knetter and G. S. Uhrig, Eur. Phys. J. B 13, 209 (2000).

[28] S. Dusuel and J. Vidal, in preparation.

[29] M. E. Fisher and M. N. Barber, Phys. Rev. Lett. 28, 1516 (1972).

[30] X. Wang and K. Mølmer, Eur. Phys. J. D 18, 385 (2002).

[31] C. M. Newman and L. S. Schulman, J. Math. Phys. 18, 23 (1977).

[32] R. H. Dicke, Phys. Rev. 93, 99 (1954). 